\title{
Automated image analysis of immunohistochemical stained brain slices of long term polyimid brain implants
}

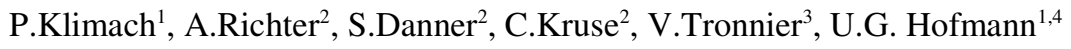 \\ 1 Institute for Signal Processing, University of Luebeck, Luebeck, Germany \\ ${ }^{2}$ Group of Cell Differentiation, Fraunhofer Research Institution for Marine Biotechnology, Fraunhofer Society, Lue- \\ beck, Germany \\ 3 Department of Neurosurgery, University of Luebeck, Luebeck, Germany \\ ${ }^{4}$ Research Group for Neuroelectronic Systems, Department of Neurosurgery, University Medical Center Freiburg, Al- \\ bert-Ludwigs-University, 79108 Freiburg, Germany, ulrich.hofmann[at]coregen.uni-luebeck.de
}

\begin{abstract}
The current quest for brain-machine-interfaces is hindered by the need to find a reliable solution for truly chronic brain implants. One approach to tackle that problem is not only to use flexible, mechanically compliant micro recording probes, but to enhance their acceptance in the target tissue by biologization. In order to shed light on the best modifications, immunohistochemical staining of long term implants in rat brains provided thousands of images over a six month experimental phase. The current work explains the image processing method, how these images were analysed in the least ambiguous way to provide meaningful and statistically relevant results. Quantitative analysis of the images allowed for the comparison of changes within the image and comparison between different images.
\end{abstract}

\section{Introduction}

Deep brain stimulation (DBS) and other invasive treatments of neurological disorders require the insertion of electrodes into appropriate target areas of the patient's brain [1]. However, insertion of any artificial device into the brain results in a complex immune response [2]. This so-called foreign body response on deep brain stimulating electrodes results at least in the establishment of glial scar tissue by reactive astrocytes [3]. Sometimes especially dense glial barriers may severely affect the electrical connection between electrode and tissue to be stimulated [4].

This effect becomes increasingly important with decreasing electrode size as approached in most recent microstimulation experiments and DBS electrodes [5, 6]. One main cause for this adversarial encapsulation is thought to be the mechanical compliance mismatch between any probe and brain tissue. Therefore several approaches are documented to develop flexible probes floating with the brain's micro motion and thus reducing mechanical friction and inflammatory responses $[7,8]$.

Unfortunately the final verdict into the chronic effects of flexible probes on brain tissue is not yet given. In order to shed light on this question we conducted long term implantation experiments in vivo, to be reported on in detail elsewhere [14]. Here we describe the image processing methods used to quantitatively analyze the resulting immunohistochemically stained brain slices.

\section{Materials and Methods}

\subsection{Immunohistochemical staining}

Immunohistochemical staining of brain slices was performed after a defined time of implantation of polyimid based flexible microprobes [10]. With this method several specific proteins, like ED1 for inflammatory response and macrophage/microglia presence, GFAP for astrocyte presence and glial scarring, or NF (neurofilament) for the presence of neurons are stained by well known, antibody based fluorescent markers [12]. Thus the brain tissue staining resulted in different colors depending on the visible type of cell. The color and intensity of a fluorescent image can thus be used to determine the quantity of a particular cell type, necessary to assess the outcome of an implantation.

In order to determine the changes in the brains of rats due to the implanted electrodes, five thousand maximal fluorescent projection images were produced using an Axio Observer Z1 Zeiss microscope.

\subsection{ImageJ for image management}

The freely available software ImageJ (NIH) [12] was chosen as the tool to extract quantitative information from the huge number of available images.

ImageJ is a Java-based image processing program which can display, edit, analyze, process, save and print many 
types of image formats. Since ImageJ is an open source program, it allows for the expansion of the software using plugins. In our case, a plugin modification of ImageJ was made for the analysis of the images by utilizing the so called ImagePlus object.

\subsection{Image preprocessing}

The Zeiss microscope produces proprietary image files in .zvi format using the RGB color space. ImageJ, however, does not natively read the .zvi format . Thus the images are transformed to .bmp files separated into their red, green and blue channels, so that the intensity of the image indicates the amount of red, green, or blue respectively. All images analyzed in this project were thus 8 bit gray scale coded, single color images and were manually mapped to their respective sizes by their magnification value. An example image of a green channel, to be analyzed, is shown in Figure 1 with a magnification of $20 \mathrm{x}$ and thus a size of 106 pixels corresponding to $100 \mu \mathrm{m}-$ which results in $0.94 \mu \mathrm{m}$ per pixel.

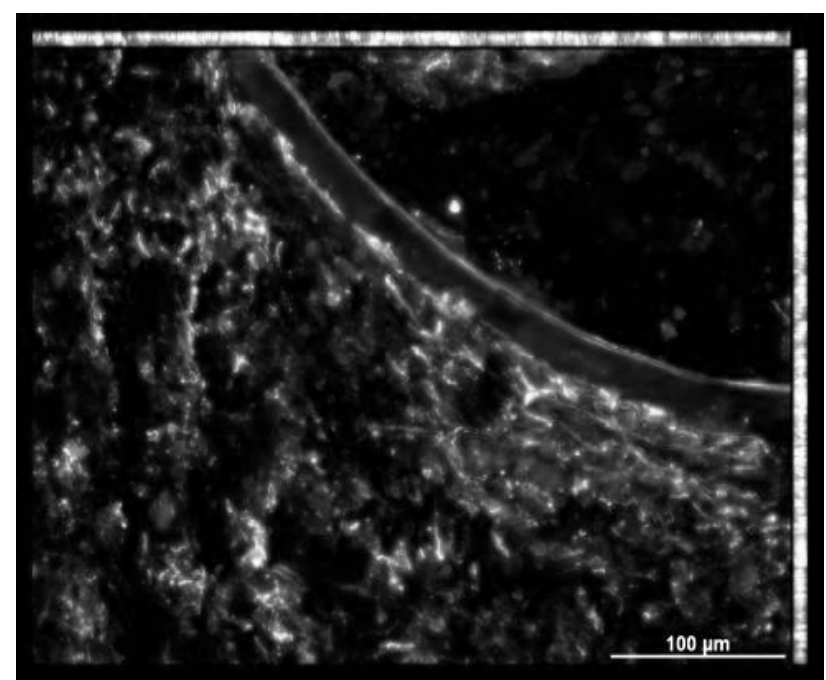

Figure 1: Example image of a brain slice using the green channel in a 20x magnification. In this slice, the green content depicts the density of markers of astrocytic cells.

\subsection{Probe location modeled as BEZIER curve}

Collected brain slice images were not fully consistent with respect to their display of the probe's cross section, since the microtome slicing and processing procedure of brain tissue may have moved or distorted the probe's location. Some probes were not visible at all, whereas some probes were fragmented, thus user input is required to select the true location. A BEZIER model is chosen to describe the precise electrode location in the image. An internal model of the otherwise fuzzy probe cross-section is thus manually created to reliably determine the distance of any pixel from the probe's edge. The corresponding BEZIER curve is defined by choosing three points at the edge of the visible probe, its two corners, $\mathrm{P}_{0}$ and $\mathrm{P}_{1}$, and a center point $\mathrm{P}_{2}$.

These points make up the control points. The curve is then given by

$$
C(t)=\sum_{i=0}^{n} P_{i} B_{i}, n
$$

where $\mathrm{B}_{\mathrm{i}, \mathrm{n}}(\mathrm{t})$ is a BERNSTEIN polynomial

$$
B(t)=(1-t)^{2} P_{0}+2(1-t) t P_{1}+t^{2} P_{2}, t \in[0,1]
$$

A BEZIER curve always passes through the first and last control points and lies inside the convex hull of the control points. The curve is tangential to $\mathrm{P}_{1}-\mathrm{P}_{0}$ and $\mathrm{P}_{\mathrm{n}}-\mathrm{P}_{(\mathrm{n}-1)}$ at the endpoints. A desirable property of BEZIER curves is that the curve is a rational curve and therefore can be translated and rotated by performing these operations on the control points only [9].

Changing a single control point changes the global shape of the curve which is easy to calculate from the control points [9]. In our case there are only three points to be defined by the user. Furthermore, it is advantageous to change the global shape of the curve by simply moving one point to find the edge along the length of the probe, as depicted in Figure 2.

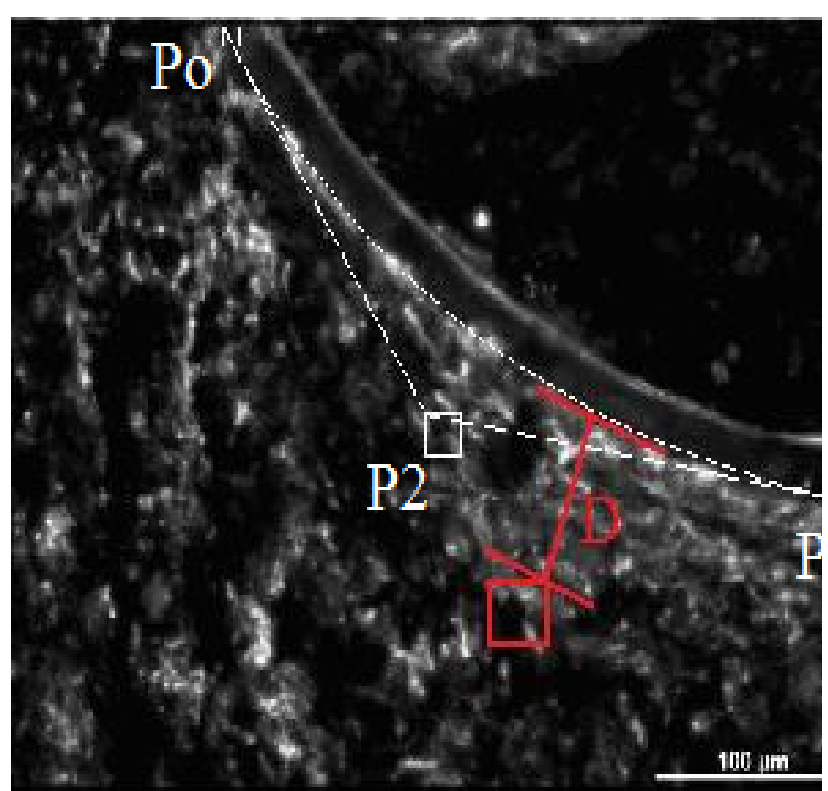

Figure 2: BEZIER curve model of the edge of the Probe with the first, second, and third point that are used to calculate the BEZIER curve, $P_{0}, P_{1}$, and $P_{2}$. The red components in the image illustrate the distance, $D$, that is from the BEZIER curve along the normal through said pixel. 


\subsection{Distance of every pixel from the probe}

The probe's BEZIER coordinates are now used to determine the distance of pixels, within the image, from the probe position modeled in the previous step. The distance is determined by finding the normal to the probe that passes through the pixel.

$$
\begin{gathered}
D^{2}=x^{2}+y^{2} \\
B(t)=(1-t) 2 P_{0}+2(1-t) t P_{1}+t^{2} P 2, t \in[0,1]
\end{gathered}
$$

Using the distance equation and the BERNSTEIN polynomial, given above, the resulting equation for the distance from the pixel to the probe is thus:

$$
\begin{gathered}
D^{2}(t)=\left[x_{p}-\left[(1-t)^{2} x_{0}+2(1-t) t x_{1}+t^{2} x_{2}\right]\right]^{2}+\left[y_{p}-\right. \\
\left.\left[(1-t)^{2} y_{0}+2(1-t) t y_{1}+t^{2} y_{2}\right]\right]^{2}
\end{gathered}
$$

where $\mathrm{D}=$ the distance

$x_{p}$ represents the $x$ coordinates of the pixel and $y_{p}$ stands for the $\mathrm{y}$ coordinates of the pixel. $\mathrm{x}_{0}, \mathrm{x}_{1}$, and $\mathrm{x}_{2}$ are the $\mathrm{x}$ coordinates of the first, second, and third point, respectively, on the BEZIER curve. $\mathrm{y}_{0}, \mathrm{y}_{1}$, and $\mathrm{y}_{2}$ are their $\mathrm{y}$ coordinates. In order to find the shortest distance to the point on the BEZIER curve, and therefore the normal from the pixel through the curve, solve for the following using NEWTON's Method:

$$
\begin{gathered}
d D / d t=0 \\
X_{n+1}=x_{n}-D\left(t_{n}\right) / D^{\prime}\left(t_{n}\right)
\end{gathered}
$$

\subsection{Divide pixel distance into distance bins}

Using the distance values calculated above, the pixels are divided into groups. All of the equidistant bins have the same width of $10 \mu \mathrm{m}$ (Figure 3 ). Each level is ordered by their distance from the electrode so that the pixels with the shortest distance from the probe are in the bins closer to the probe.

Due to the implantation method [10], the area of interest is the convex side of the flexible probe. At this point, there are two values created, the distance from the probe in relation to the other pixels, and the intensity of the color at this distance.

\section{Results and Discussion}

The introduced semi-automatic analysis program, based on ImageJ and simple image processing methods, is very much able to perform semi-automated analysis of fluorescent images with a high throughput.

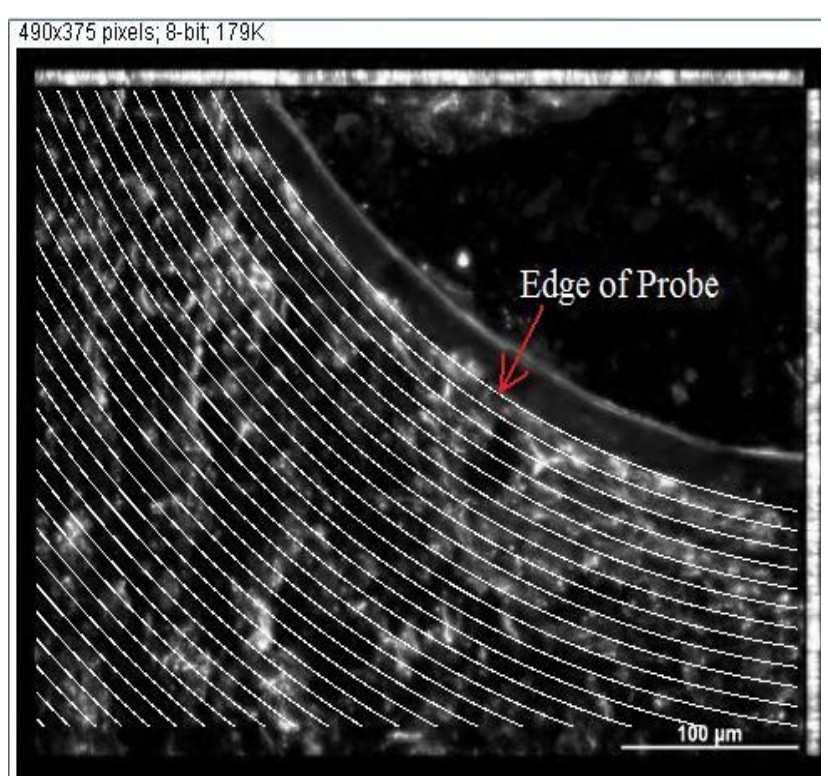

Figure 3: Illustrates the equidistant bins of equal width that divided the pixels of the image by the distance form the probe. Each bin had a width of $10 \mu \mathrm{m}$

Analyzing the fluorescence intensity of acquired images, with respect to the distance from the implanted probe, allows us to determine the density of immunostained proteins. This gives unambiguous, quantitative answers about the comparison of the body's response to different probe types or its response in different anatomical regions over a long period of time.

Figure 4 depicts a graph of the distance-binned fluorescence distribution for the glial scar marker GFAP in an exemplary slice after 6 weeks of exposure to a polyimid probe in the rat cortex. The Gaussian mean of the fluorescence intensity for the pixels in each bin are calculated. This mean is plotted against the distance of the pixel from the probe. The distance of the bin was marked between the edge of the bin, closest to the probe, to the edge of the probe described by the BEZIER curve.

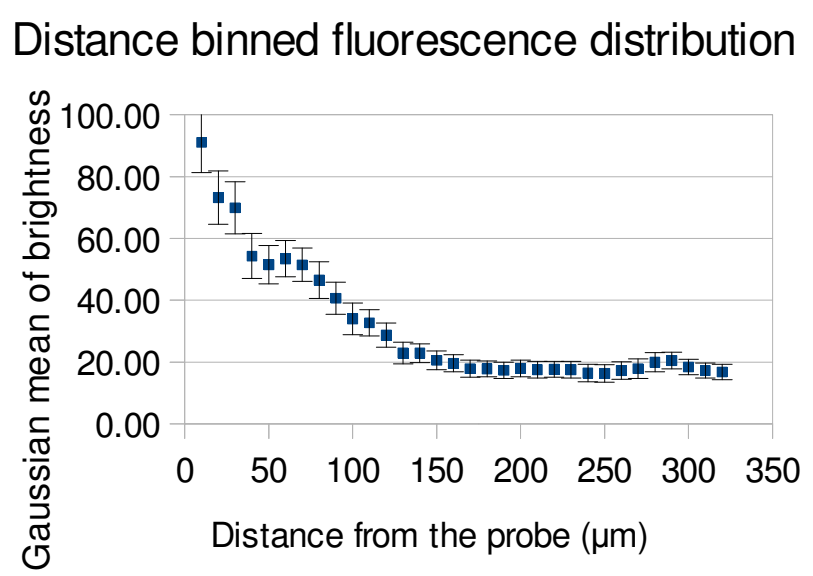

Figure 4: Fluoresence intensity of images in relation to the distance from the electrode 
Illustrated in Figure 5 is the development of the GFAP intensity from one week to six weeks implantation. This shows the body's reaction towards this polymid device.

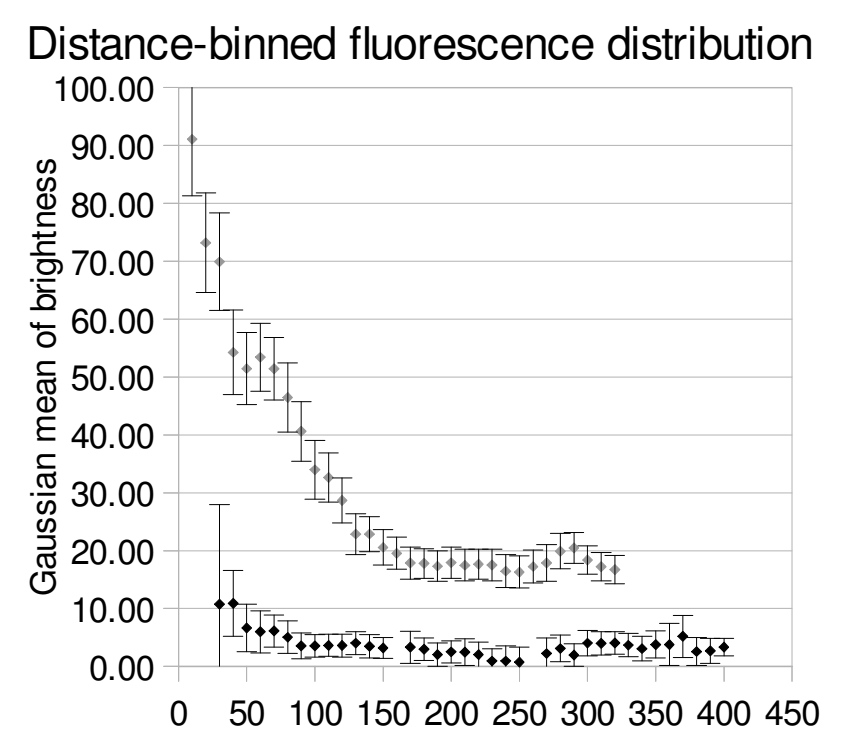

Distance from probe surface $(\mu \mathrm{m})$

Figure 5: The difference between the GFAP intensity after 1 week and that after 6 weeks is clearly shown by the difference in the binned fluorescence, where the result after 6 weeks, light gray diamonds icon, is comparatively higher than the results after a week, black diamonds icons.

\section{Conclusion}

In the pursuit of the knowledge of the chronic effects of flexible probes on brain tissue, hundreds of images from immunohistochemical staining were created. The images displayed had different colors which were used to determine the density and types of cells. The image analysis procedure described above provides an objective and unambiguous method to compare these images. As part of the image analysis the flexible probe needed to be identified - best done manually. The described image analysis program supports the procedure with a semiautomated method of determining the position of the probe. Once the position of the flexible probe has been determined then the image is divided into bins of the same width and the average of the color value of the pixels within each bin is calculated. The result is a value for the color intensity with a relative distance to the flexible probe. These resulting values provide a means to compare one image to another image and one immuno label's result to another. The final result provides an indication of the body's reaction to the probe (to be presented elsewhere) [14].

\section{References}

1. Tronnier, V. M., W. Fogel, et al. (2002). "High frequency stimulation of the basal ganglia for the treatment of movement disorders: current status and clinical results." Minim. Invasive Neurosurg. 45: 91-96.

[2] Reichert, W. M., Ed. (2008). Indwelling Neural Implants. Frontiers in Neuroengineering. Boca Raton, FL, CRC Press.

[3] Sun, D. A., H. Yu, et al. (2008). "Postmortem analysis following 71 months of deep brain stimulation of the subthalamic nucleus for Parkinson disease." J. Neurosurg. 109(325-329).

[4] Polikov, V. S., P. A. Tresco, et al. (2005). "Response of brain tissue to chronically implanted neural electrodes “ J Neurosci Methods

[5] Hiller, A., S. Loeffler, et al. (2007). "Electrical High Frequency Stimulation Induces GABA Outflow in Freely Moving Rats.” J. Neurosci. Methods 159(2): 286-290.

[6] Martens, H. C. F., E. Toader, et al. (2010). "Spatial steering of deepbrain stimulation volumes using a novel lead design." Clinical Neurophysiology.

[7] Stieglitz, T. and J. U. Meyer (1999). "Implantable microsystems. Polyimide-based neuroprostheses for interfacing nerves." Med. Device Technol. 10: 28-30.

[8] Rousche, P. J., D. S. Pellinen, et al. (2001). "Flexible Polyimide-based intracortical electrode arrays with bioactive capability." IEEE Trans Biomed Eng 48: 361371.

[9] Foley; Computer Graphics: Principles and Practice; 1996

[10] Xie, Y., S. Löffler, et al. (2010). A novel approach of flexible electrode implantation for deep brain recording. Biomed Tech. O. Dössel. Rostock, Walter de Gruyter. 55 (Supp 1): 4.

[11] Richter, A., Y. Xie, et al. (2012). "Easy adaptable implantation method for flexible deep brain stimulating electrodes to rat brains." $\mathrm{J}$ of Visualized Experiments submitted 04/2012.

[12] Biran, R., D. C. Martin, et al. (2007). "The brain tissue response to implanted silicon microelectrode arrays is increased when the device is tethered to the skull." Journal of Biomedical Materials Research Part A

[13] NIH; http://rsbweb.nih.gov/ij/docs/intro.html

[14] Richter, A. (2012). Biologisierung Tiefenhirn-stimulierender Implantate durch Besiedelung mit adulten glandulären Stammzellen. Section iof Science and Technology. Lübeck, University of Lübeck. 